\title{
$\beta$-Fluorofentanyls Are pH-Sensitive Mu Opioid Receptor Agonists
}

\author{
Ricardo Rosas, Jr., Xi-Ping Huang, ${ }^{\dagger}$ Bryan L. Roth, ${ }^{\ddagger}{ }^{\dagger}\left({ }_{0}\right.$ and Chris Dockendorff* ${ }^{\dagger}$ (i) \\ ${ }^{\dagger}$ Department of Chemistry, Marquette University, P.O. Box 1881, Milwaukee, Wisconsin 53201-1881, United States \\ ${ }^{\ddagger}$ National Institute of Mental Health Psychoactive Drug Screening Program, Department of Pharmacology, School of Medicine, \\ University of North Carolina Chapel Hill, Chapel Hill, North Carolina 27599-7365, United States \\ \# Division of Chemical Biology and Medicinal Chemistry, Eshelman School of Pharmacy, University of North Carolina Chapel Hill, \\ Chapel Hill, North Carolina 27599-7365, United States
}

\begin{abstract}
The concept recently postulated by Stein and co-workers (Science 2017, 355, 966) that mu opioid receptor (MOR) agonists possessing amines with attenuated basicity show $\mathrm{pH}$-dependent activity and can selectively act at damaged, low $\mathrm{pH}$ tissues has been additionally supported by in vitro studies reported here. We synthesized and tested analogs of fentanyl possessing one or two fluorine atoms at the beta position of the phenethylamine side chain, with additional fluorines optionally added to the benzene ring of the side chain. These compounds were synthesized in 1 to 3 steps from
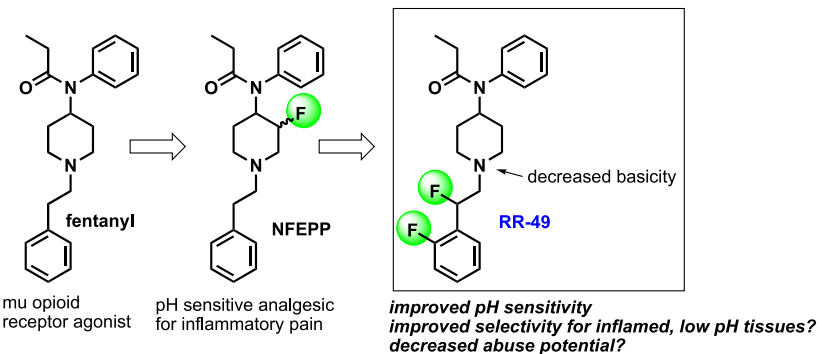
commercial building blocks. The novel bis-fluorinated analog RR-49 showed superior $\mathrm{pH}$ sensitivity, with full efficacy relative to DAMGO, but with 19-fold higher potency (IC 50 ) in a MOR cAMP assay at $\mathrm{pH} 6.5$ versus 7.4. Such compounds hold significant promise as analgesics for inflammatory pain with reduced abuse potential.
\end{abstract}

KEYWORDS: $M u$ opioid receptor agonist, fentanyl, fluorination, $p H$-sensitive, analgesic, cAMP

A gonism of the mu opioid receptor (MOR), originally via the natural product morphine, has been utilized for millennia as the most effective form of analgesia for acute pain. ${ }^{1}$ The potential for addiction and abuse with sustained use of narcotic MOR agonists, combined with the dangerous side effect of respiratory depression, has driven the present opioid epidemic, with more than 70,000 overdose deaths in the U.S. in 2017. ${ }^{2}$ Fortunately, new approaches to MOR agonism have been investigated that could lead to analgesics with improved safety profiles. $^{3-7}$ Stein and co-workers have recently added an additional strategy worthy of careful consideration: the use of $\mathrm{pH}$-dependent ligands designed to have a higher affinity for receptors in damaged tissues with lower $\mathrm{pH}$. This strategy leverages the fact that a protonated amine is generally required in MOR agonists to form an ion pair with Asp147 of human MOR, as suggested by site-directed mutagenesis ${ }^{8}$ and confirmed by the X-ray structure of BU72 bound to MOR. ${ }^{9}$ A fluorinated version of fentany $1^{10-12}(\mathbf{1}$, Chart 1$)$ called $( \pm)-N$-(3-fluoro-1phenethylpiperidine-4-yl)- $N$-phenyl propionamide (NFEPP) (2), was reported by Stein et al. to have increased affinity for MOR and activity in functional $\mathrm{G}_{\mathrm{i}}$-driven assays at $\mathrm{pH} 6.5$ versus normal $\mathrm{pH}(7.4) .{ }^{13}$ Notably, NFEPP was also reported to be effective in rat models of acute and/or persistent inflammatory pain and may have lower CNS-related side effects than fentanyl. ${ }^{13,14}$ The relative stereochemistry of this compound was not disclosed.
Chart 1. Fentanyl and Fluorinated Analogs
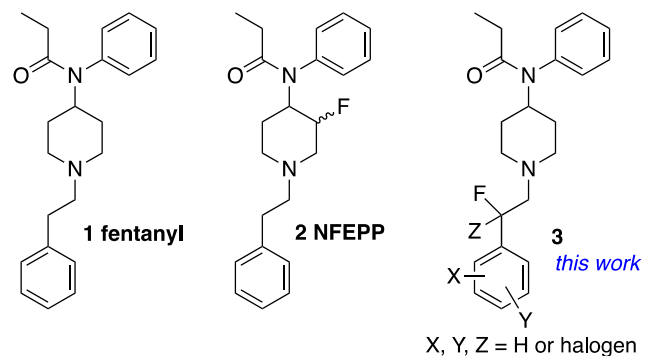

We reasoned that other MOR ligands with carefully attenuated basicities could be effective peripheral analgesics; such compounds (3, Chart 1 ) could be easier to prepare than NFEPP, are easily accessible in pure isomeric forms, and can be prepared via flexible synthetic routes that offer the opportunity to identify analogs with superior $\mathrm{pH}$ sensitivity and drug-like properties. The potential to selectively activate MORs at damaged peripheral sites at lower $\mathrm{pH}$, over central MORs known to mediate undesirable effects such as euphoria and respiratory depression, makes this approach worthy of further investigation.

Received: July 24, 2019

Accepted: August 13, 2019

Published: August 13, 2019 
The introduction of $\mathrm{p} K_{\mathrm{a}}$-lowering electronegative fluorine atoms beta to amines is a well appreciated tactic that has been frequently used to improve drug-like properties. ${ }^{15-17} \mathrm{We}$ continued with this strategy applied to the fentanyl scaffold by aiming to place fluorine(s) beta to the piperidine nitrogen, but on the phenethyl side chain, rather than on the piperidine itself, as in NFEPP (Chart 1). We synthesized $\beta$-fluorofentanyl 6a and $\beta, \beta$-difluorofentanyl $\mathbf{6 b}$ via simple substitution reactions between the commercially available phenethyl halides $\mathbf{4 a}$ and $\mathbf{4 b}$ and piperidine $\mathbf{5}$ (Scheme 1 ). These analogs were compared

\section{Scheme 1. One-Step Synthesis of $\beta$-Fluorofentanyls}

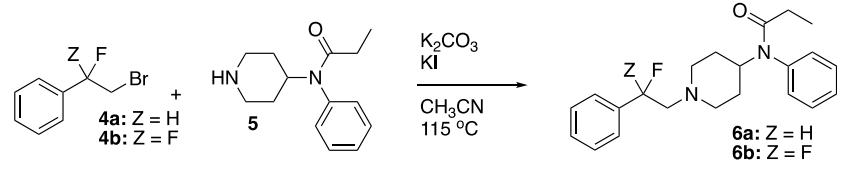

to fentanyl and NFEPP in an MOR assay in transfected HEK 293 cells measuring relative cAMP concentrations. In this assay, MOR agonists induce $\mathrm{G}_{\mathrm{i}}$-mediated inhibition of adenylyl cyclase, and the resulting changes in cAMP concentration are measured indirectly via changes in luminescence from a luciferase enzyme engineered with a cAMP binding region. ${ }^{18}$

In this assay, NFEPP and 6a performed nearly identically, with both acting as full agonists (data not shown) relative to fentanyl, both with $\mathrm{IC}_{50} \mathrm{~s}$ of $0.71 \mathrm{nM}$ at $\mathrm{pH}$ 6.5. As a measure of $\mathrm{pH}$ sensitivity, we define the "pH ratio", which is simply the ratio of $\mathrm{IC}_{50} \mathrm{~s}$ at $\mathrm{pH} 7.4$ and 6.5. Importantly, both NFEPP and 6a showed significantly higher potencies at $\mathrm{pH} 6.5$ than 7.4 , with $\mathrm{pH}$ ratios of 10.6 and 8.2, respectively, compared to fentanyl with a measured $\mathrm{pH}$ ratio of 1.9 (Figure 1 and Table 1 ). The bisfluorinated compound $\mathbf{6 b}$, with a calculated $\mathrm{p} K_{\mathrm{a}}$ of 5.5 , was expected to be only weakly active even at low $\mathrm{pH}$, and this was consistent with the experimental results, with an $\mathrm{IC}_{50}$ nearly 4 orders of magnitude higher than $\mathbf{6 a}$ (Table 1). During the course of this work, Stein reported in vitro and in vivo analgesia data with 6a (aka FF3), though details of its synthesis and characterization were not disclosed. A pH ratio was reported to be 4.9 for $6 \mathbf{a}$ in an MOR GTP $\gamma$ S assay. ${ }^{1}$

Stein reported that $\mathbf{6 a}$ showed impressive dose-dependent analgesia in rat models of inflammatory pain, though of limited duration. Unfortunately, it also showed similar conditioned place preference to fentanyl, suggesting that it could suffer from similar abuse liabilities, ${ }^{19}$ which is likely also an issue with NFEPP. We reasoned that molecules with $\mathrm{p} K_{\mathrm{a}} \mathrm{s}$ less than $6 \mathrm{a}$ (measured by Stein to be 7.22$)^{19}$ but greater than $\mathbf{6 b}$ (calculated to be 5.48 , Table 1) could offer substantially better selectivity for inflamed tissues and a larger therapeutic window.

With these promising results in hand for $6 \mathrm{a}$, we aimed to prepare molecules with amine $\mathrm{p} K_{\mathrm{a}} \mathrm{s}$ closer to 6.5 , i.e., the $\mathrm{pH}$ that may be present in inflamed tissues. To do this, we simply added fluorine(s) to the benzene of the phenethyl side chain. Since the required alkyl halides were not commercially available, we pursued alternative routes that could also support the preparation of enantiopure materials, if desired. Since styrene oxides are potentially available in highly enantioenriched forms via asymmetric epoxidation methods, we first attempted epoxide opening reactions with piperidine 5, with alcohol products that could be subsequently converted to the desired fluorides in a single step.

Somewhat unexpectedly, the ring opening reactions under basic conditions yielded regioisomeric products that were
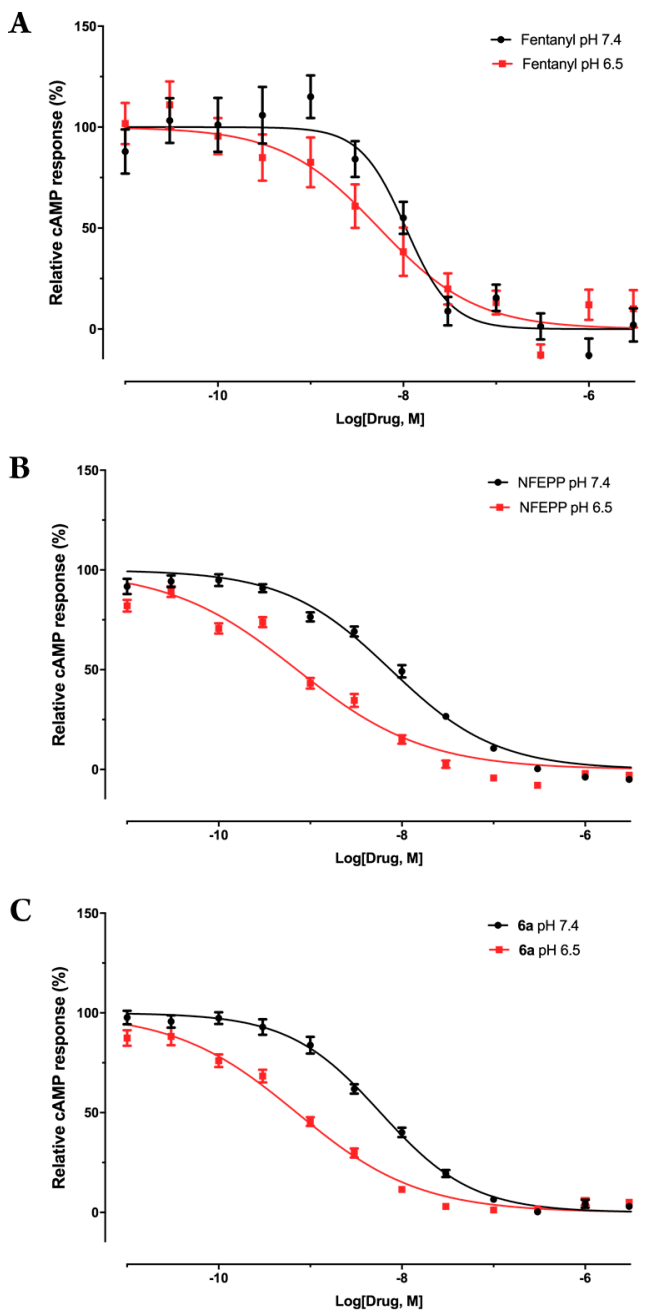

Figure 1. Concentration-response curves of (A) fentanyl; (B) NFEPP; and (C) 6a in GloSensor MOR cAMP assay. Data was individually normalized to each compound to show \% response.

Table 1. Summary of MOR Agonist Activities ${ }^{a}$

\begin{tabular}{llccc}
\multicolumn{1}{c}{ compound } & $\begin{array}{c}\mathrm{calcd} \mathrm{pK}^{b} \\
(\text { exptl })\end{array}$ & $\begin{array}{c}\mathrm{pIC}_{50} \\
(\mathrm{pH} \mathrm{7.4})^{c}\end{array}$ & $\begin{array}{c}\mathrm{pIC}_{50} \\
(\mathrm{pH} \mathrm{6.5})^{c}\end{array}$ & $\begin{array}{c}\mathrm{pH} \\
\text { ratio }^{d}\end{array}$ \\
DAMGO & & $9.10 \pm 0.07$ & $8.71 \pm 0.07$ & 0.4 \\
fentanyl (1) & $8.77(8.4)$ & $7.97 \pm 0.08$ & $8.26 \pm 0.12$ & 1.9 \\
NFEPP (2) & $7.30(6.82)$ & $8.12 \pm 0.03$ & $9.15 \pm 0.04$ & 10.6 \\
6a & $7.18(7.22)$ & $8.23 \pm 0.03$ & $9.15 \pm 0.04$ & 8.2 \\
6b & 5.48 & $4.50 \pm 0.07$ & $5.24 \pm 0.06$ & 5.4 \\
RR-49 (12a) & 6.60 & $6.98 \pm 0.07$ & $8.26 \pm 0.07$ & 19.0 \\
12b & 6.64 & $7.11 \pm 0.09$ & $8.19 \pm 0.07$ & 12.0 \\
12c & 6.94 & $6.99 \pm 0.07$ & $7.60 \pm 0.06$ & 4.0 \\
12d & 6.38 & $7.05 \pm 0.11$ & $7.93 \pm 0.07$ & 9.0
\end{tabular}

${ }^{a}$ Agonist activities were measured with a GloSensor cAMP assay with HEK 293 cells in 384-well plates transiently expressing MOR. See Supporting Info for full details. Results were independently normalized, with $0 \%$ and $100 \%$ activity defined for each compound as the top and bottom of curves fit with 4-parameter nonlinear regression (GraphPad Prism v. 8). ${ }^{b} \mathrm{p} K_{\mathrm{a}}$ of protonated amine calculated with ChemAxon Marvin v.18.3. ${ }^{c} \mathrm{pIC}_{50}=-\log \left(\mathrm{IC}_{50}\right)$. Uncertainty is indicated by SEM for the curve fitting to a minimum of 16 measurements. ${ }^{d} \mathrm{pH}$ ratio $=\mathrm{IC}_{50}(\mathrm{pH} 7.4) / \mathrm{IC}_{50}(\mathrm{pH} \mathrm{6.5})$.

surprisingly difficult to separate. For example, treatment of epoxide 7 with 5 generated a mixture of the desired secondary 
alcohol 8a and undesired primary alcohol 9a (Scheme 2). Alternatively, piperidine 5 was cleanly alkylated with $\alpha$ -

Scheme 2. Epoxide Opening Reaction

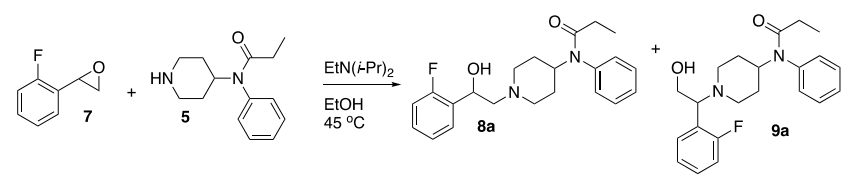

bromoacetophenones 10, then the resulting ketones 11 reduced with sodium borohydride to generate the desired amino alcohols 8 (Scheme 3). Finally, these were converted to the final $\beta$ -

Scheme 3. Three-Step Synthesis of $\beta$-Fluorofentanyls
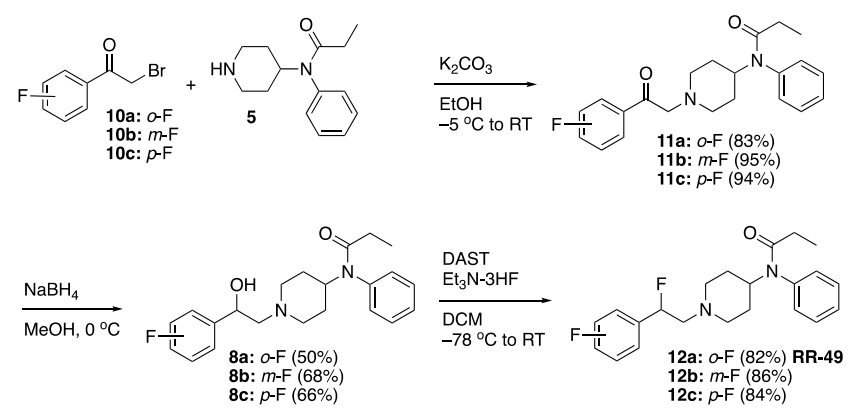

fluorofentanyl analogs 12 by treatment with both DAST and $\mathrm{Et}_{3} \mathrm{~N}-3 \mathrm{HF}$, which presumably protonates the basic amine to minimize formation of fluoroamine byproducts. The 2,5difluorophenyl analog 12d was generated without isolation of the intermediate ketone $1 \mathbf{1 d}$, which was particularly unstable (Scheme 4).

\section{Scheme 4. Synthesis of $12 d$}
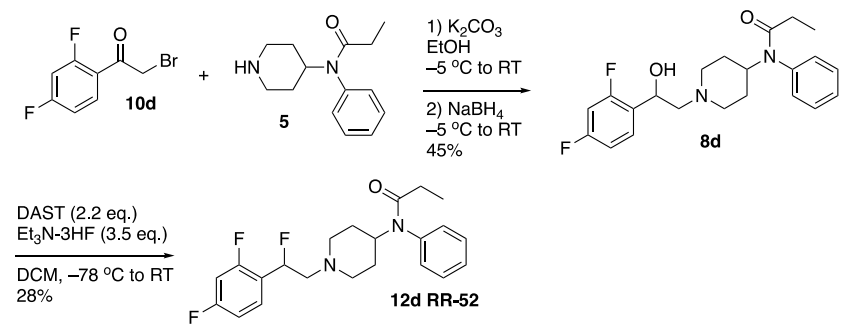

The analogs $12 \mathbf{a}-\mathbf{d}$ were tested in the GloSensor assay along with the standard MOR agonist DAMGO and fentanyl (Figure 2; Table 1). As expected, the $\mathrm{pH}$ ratio tracks with the amine $\mathrm{p} K_{a}$ as the ortho-fluoro analog 12a (called RR-49), with its lower $\mathrm{p} K_{\mathrm{a}}$ value of 6.60 , showed better $\mathrm{pH}$ sensitivity $(\mathrm{pH}$ ratio $=19.0$, Figure 3$)$ relative to the para-fluoro $12 \mathrm{c}\left(\right.$ calcd $\mathrm{p} K_{\mathrm{a}}=6.94 ; \mathrm{pH}$ ratio $=4.0)$. However, the 2,4-difluoro analog $12 \mathrm{~d}$ is the exception to the trend, as it had a lower $\mathrm{pH}$ ratio than $12 \mathrm{a}$ (9.0). We cannot rule out the fact that a fluorine in the para position may have additional effects on receptor activation.

In summary, we have identified 12a (RR-49) as a more highly $\mathrm{pH}$-sensitive MOR agonist than the prior reported compounds NFEPP (2) and 6a. Compound 12a also maintains full agonist activity relative to DAMGO and fentanyl, as estimated in Figure 2. In vivo studies are underway to confirm that it may impart lower CNS effects and abuse liabilities than prior compounds of this class. $\mathrm{pH}$-sensitive MOR agonists may represent a
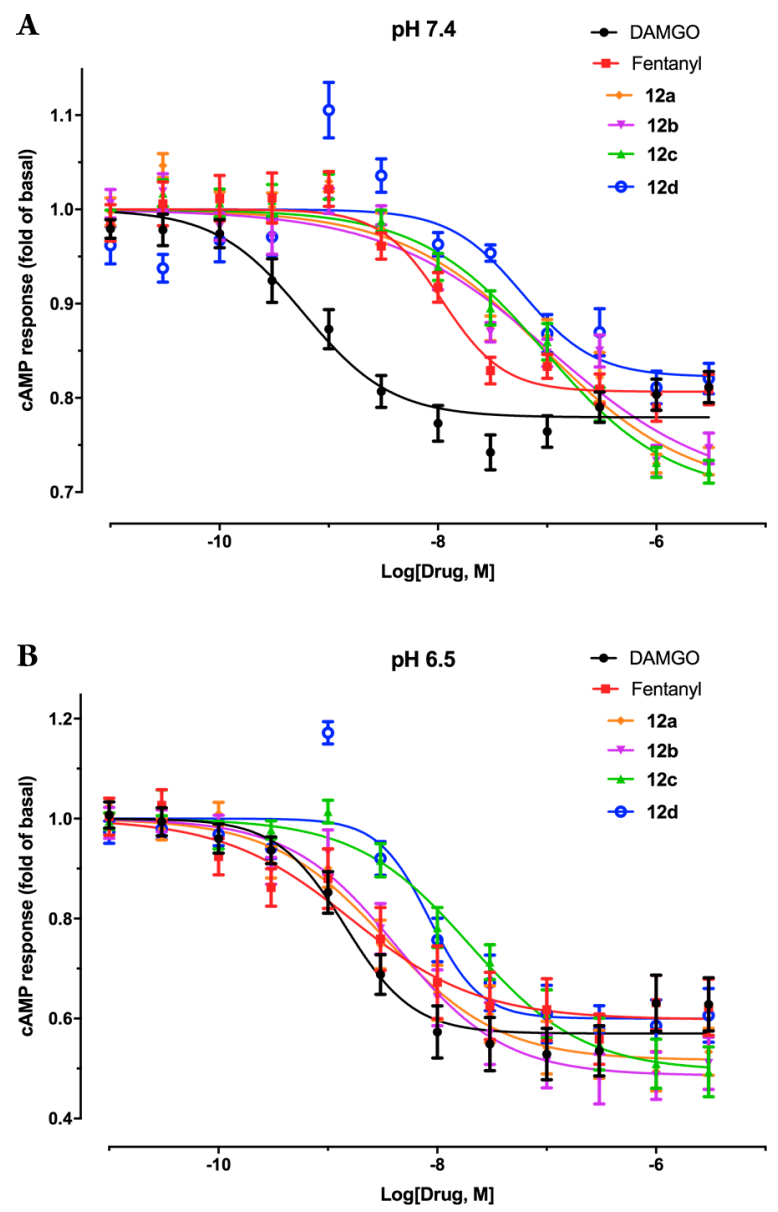

Figure 2. Concentration-response curves (fold activity) of DAMGO, fentanyl, and agonists 12a-d in the GloSensor MOR cAMP assay at $\mathrm{pH}$ 7.4 (A) and 6.5 (B). Results were normalized with basal activity as 1.0 and analyzed in Prism using the built-in 4-parameter logistic function.

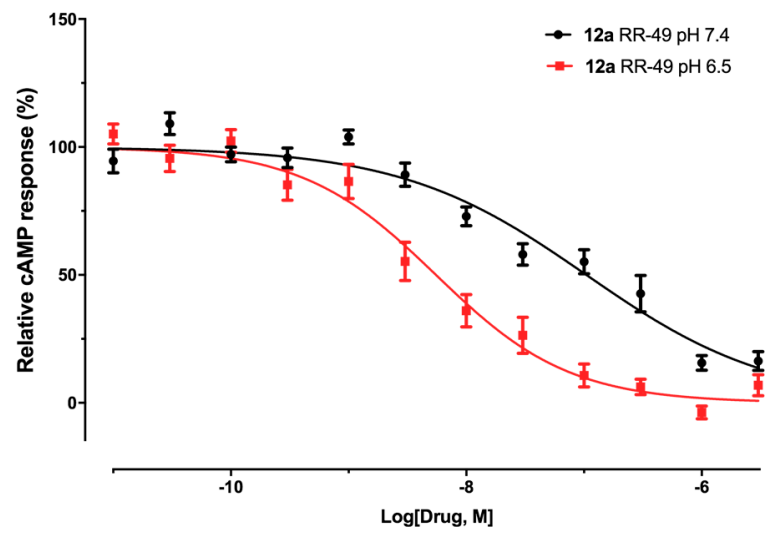

Figure 3. pH-dependent activity of 12a (RR-49) (\% response).

promising strategy for the treatment of inflammatory pain with decreased risks relative to current opioids.

\section{ASSOCIATED CONTENT}

\section{S Supporting Information}

The Supporting Information is available free of charge on the ACS Publications website at DOI: 10.1021/acsmedchemlett.9b00335. 
MOR cAMP assay protocol; synthetic protocols; characterization data $\left({ }^{1} \mathrm{H} \mathrm{NMR},{ }^{13} \mathrm{C} \mathrm{NMR}\right.$, and LCMS chromatograms) (PDF)

\section{AUTHOR INFORMATION}

\section{Corresponding Author}

*E-mail: christopher.dockendorff@mu.edu. Tel: 1-414-2881617.

ORCID

Bryan L. Roth: 0000-0002-0561-6520

Chris Dockendorff: 0000-0002-4092-5636

\section{Author Contributions}

Designed compounds: C.D. Developed synthetic routes: C.D., R.R. Synthesized and characterized compounds: R.R. Performed assays: X.-P.H. Processed and analyzed data: X.-P.H., B.L.R., C.D. Wrote the manuscript: C.D. Prepared Supporting Info: R.R., C.D. Edited the manuscript: X.-P.H., B.L.R.

\section{Funding}

R.R. and C.D. thank Marquette University for funding. B.L.R. and X.-P.H. were supported by the NIMH Psychoactive Drug Screening Program and P01DA035764.

\section{Notes}

An earlier version of this manuscript was submitted to the preprint server ChemRxiv. ${ }^{20}$

The authors declare the following competing financial interest(s): A patent application including this work has been submitted with C.D. and R.R. as inventors.

\section{ACKNOWLEDGMENTS}

We thank Dr. Christoph Stein (Charité Universitätsmedizin Berlin) for generously providing a sample of NFEPP for in vitro studies. We thank Dr. Sheng Cai (Marquette University) for assistance with LC-MS and NMR instruments.

\section{ABBREVIATIONS}

cAMP, cyclic adenosine monophosphate; CNS, central nervous system; DAMGO, H-Tyr-D-Ala-Gly-N-MePhe-Gly-ol; DAST, diethylaminosulfur trifluoride; DCM, dichloromethane; DIEA, $\mathrm{N}, \mathrm{N}$-diisopropylethylamine; GPCR, G-protein coupled receptor; HEK, human embryonic kidney; $\mathrm{IC}_{50}$, half-maximal inhibitory concentration; MOR, mu opioid receptor; SEM, standard error of the mean

\section{REFERENCES}

(1) Pasternak, G. W.; Pan, Y.-X. Mu Opioids and Their Receptors: Evolution of a Concept. Pharmacol. Rev. 2013, 65, 1257-1317.

(2) U.S. Centers for Disease Control and Prevention. https://wonder. cdc.gov/.

(3) Siuda, E. R.; Carr, R.; Rominger, D. H.; Violin, J. D. Biased MuOpioid Receptor Ligands: a Promising New Generation of Pain Therapeutics. Curr. Opin. Pharmacol. 2017, 32, 77-84.

(4) Yekkirala, A. S.; Roberson, D. P.; Bean, B. P.; Woolf, C. J. Breaking Barriers to Novel Analgesic Drug Development. Nat. Rev. Drug Discovery 2017, 16, 545-564.

(5) Chan, H. C. S.; McCarthy, D.; Li, J.; Palczewski, K.; Yuan, S. Designing Safer Analgesics via M-Opioid Receptor Pathways. Trends Pharmacol. Sci. 2017, 38, 1016-1037.

(6) Cunningham, C. W.; Elballa, W. M.; Vold, S. U. Bifunctional Opioid Receptor Ligands as Novel Analgesics. Neuropharmacology 2019, 151, 195-207.

(7) Roth, B. L. Molecular Pharmacology of Metabotropic Receptors Targeted by Neuropsychiatric Drugs. Nat. Struct. Mol. Biol. 2019, 26, 535-544.
(8) Surratt, C. K.; Johnson, P. S.; Moriwaki, A.; Seidleck, B. K.; Blaschak, C. J.; Wang, J. B.; Uhl, G. R. Mu Opiate Receptor. Charged Transmembrane Domain Amino Acids Are Critical for Agonist Recognition and Intrinsic Activity. J. Biol. Chem. 1994, 269, 2054820553.

(9) Huang, W.; Manglik, A.; Venkatakrishnan, A. J.; Laeremans, T.; Feinberg, E. N.; Sanborn, A. L.; Kato, H. E.; Livingston, K. E.; Thorsen, T. S.; Kling, R. C.; Granier, S.; Gmeiner, P.; Husbands, S. M.; Traynor, J. R.; Weis, W. I.; Steyaert, J.; Dror, R. O.; Kobilka, B. K. Structural Insights Into M-Opioid Receptor Activation. Nature 2015, 524, 315321.

(10) Janssen, P. A. A. Review of the Chemical Features Associated with Strong Morphine-Like Activity. Br. J. Anaesth. 1962, 34, 260-268.

(11) Janssen, P. A. J.; Gardocki, J. F. Method for Producing Analgesia. U.S. $3,141,823$.

(12) Vardanyan, R. S.; Hruby, V. J. Fentanyl-Related Compounds and Derivatives: Current Status and Future Prospects for Pharmaceutical Applications. Future Med. Chem. 2014, 6, 385-412.

(13) Spahn, V.; Del Vecchio, G.; Labuz, D.; Rodriguez-Gaztelumendi, A.; Massaly, N.; Temp, J.; Durmaz, V.; Sabri, P.; Reidelbach, M.; Machelska, H.; Weber, M.; Stein, C. A Nontoxic Pain Killer Designed by Modeling of Pathological Receptor Conformations. Science 2017, 355, 966-969.

(14) Rodriguez-Gaztelumendi, A.; Spahn, V.; Labuz, D.; Machelska, H.; Stein, C. Analgesic Effects of a Novel pH-Dependent M-Opioid Receptor Agonist in Models of Neuropathic and Abdominal Pain. Pain 2018, 159, 2277-2284.

(15) Müller, K.; Faeh, C.; Diederich, F. Fluorine in Pharmaceuticals: Looking Beyond Intuition. Science 2007, 317, 1881-1886.

(16) Hagmann, W. K. The Many Roles for Fluorine in Medicinal Chemistry. J. Med. Chem. 2008, 51, 4359-4369.

(17) Gillis, E. P.; Eastman, K. J.; Hill, M. D.; Donnelly, D. J.; Meanwell, N. A. Applications of Fluorine in Medicinal Chemistry. J. Med. Chem. 2015, 58, 8315-8359.

(18) Binkowski, B. F.; Butler, B. L.; Stecha, P. F.; Eggers, C. T.; Otto P.; Zimmerman, K.; Vidugiris, G.; Wood, M. G.; Encell, L. P.; Fan, F.; Wood, K. V. A Luminescent Biosensor with Increased Dynamic Range for Intracellular cAMP. ACS Chem. Biol. 2011, 6, 1193-1197.

(19) Spahn, V.; Del Vecchio, G.; Rodriguez-Gaztelumendi, A.; Temp, J.; Labuz, D.; Kloner, M.; Reidelbach, M.; Machelska, H.; Weber, M.; Stein, C. Opioid Receptor Signaling, Analgesic and Side Effects Induced by a Computationally Designed pH-Dependent Agonist. Sci. Rep. 2018, 8,8965 .

(20) Rosas, R., Jr.; Huang, X.-P.; Roth, B. L.; Dockendorff, C. BFluorofentanyls Are $\mathrm{pH}$-Sensitive $\mathrm{Mu}$ Opioid Receptor Agonists. ChemRxiv 2019, DOI: 10.26434/chemrxiv.9036752.v1. 